\title{
Seroprevalence and exposure to risk factors for leptospirosis among veterinary students at Massey University
}

\author{
F Fang ${ }^{*}$, J Benschop*, PR Wilson*, JM Collins-Emerson*, C Heuer* and D Prattley* \\ * Institute of Veterinary, Animal and Biomedical Sciences, Massey University, Private Bag 11222, Palmerston North 4442, New \\ Zealand \\ ${ }^{\S}$ Author for correspondence. Email: f.fang1@ massey.ac.nz
}

\section{Supplementary Table 1. Particiant questionnaire: Study of Leptospirosis among veterinary students}

EpiCentre
Institute of Veterinary, Animal and
Biomedical Sciences
Private Bag 11222
Palmerston North
New Zealand
Telephone: +64 (6) 3569099 ext 81209
Facsimile: +64 (6) 3505716
www.massey.ac.nz

\section{Study of Leptospirosis among Veterinary Students Participant Questionnaire}

The objectives of this study are to measure the prevalence of leptospirosis infection or exposure in veterinary students at Massey University, and to investigate risk factors for sero-prevalence during both scheduled study and other times. This study will quantify the risk of infection, identify sources of infection, and guide the faculty in developing control measures if appropriate.

In New Zealand, leptospirosis is the most common occupationally acquired zoonotic disease. Cattle, sheep and deer are the most important animal reservoirs. Human exposure to Leptospira is mainly through contacting infected animal urine via abrasions or cuts in the skin, or intact mucous membranes. The majority of notified leptospirosis cases in New Zealand are due to occupational risk factors.

Personal information included in the questionnaire will be treated in the strictest confidence and will not be published or disclosed to any third parties by the research team in a manner that would allow identification of participants.

The study has the approval of the Massey University Human Research Ethics Committee.

The research team appreciates your participation in this study of leptospirosis.

The content of this supplementary information has not been edited. All risk and liability rest with the authors. 


\section{Participant identification}

Note: please make sure you have read through and signed the consent and confidentiality form before taking part in this study.

\begin{tabular}{|c|c|}
\hline Name & \\
\hline Gender & Female $\square$ \\
\hline Date of Birth & (day/month/year) \\
\hline $\begin{array}{l}\text { Which option best identifies your ethnic } \\
\text { affiliation? }\end{array}$ & $\begin{array}{lrr}\text { NZ-Maori } \square & \text { Pacific Islander } \square & \text { NZ-European } \square \\
\text { Asian } \square & \text { Other } \square\end{array}$ \\
\hline $\begin{array}{l}\text { Which year of the course are you currently } \\
\text { in? }\end{array}$ & $\begin{array}{l}\text { First } \square \quad \text { Second } \square \quad \text { Third } \square \\
\text { Fourth } \square \quad \text { Fifth } \square \\
\text { * If you had repeated studying for your courses, please state } \\
\quad \text { year }\end{array}$ \\
\hline Track (Fifth year students only) & $\begin{array}{l}\text { Companion Animal } \square \text { Production Animal } \square \text { Equine } \square \\
\text { Wildlife Animal } \square \quad \text { Avian Species } \square \quad \text { Mixed } \square \\
\text { Other } \square \text { (Please state) }\end{array}$ \\
\hline $\begin{array}{l}\text { What location/s have you lived in during } \\
\text { the past } 18 \text { months? } \\
\text { (please state the proportions if you have been } \\
\text { living in more than one type of the locations) }\end{array}$ & $\begin{array}{l}\text { Rural } \square \% \quad \text { Urban } \square \% \quad \text { Lifestyle* }^{*} \square \% \\
\text { * i.e. Small block with grazing animals }\end{array}$ \\
\hline
\end{tabular}




\section{Exposure within the veterinary curriculum *}

(* Includes scheduled semester courses, vacation veterinary practical requirement, and farm

practical as part of the veterinary curriculum only (other potential exposures are in section 3 below))

This section asks about your potential exposure to the risk of leptospirosis from each of the animal species you may have been in contact with during the veterinary curriculum.

\subsection{Appliance of personal protective equipment during veterinary curriculum}

During the veterinary curriculum, approximately what percentage of the time do you use the recommended personal protective equipment, such as gloves, overalls, face mask and/or goggles? $\%$

\subsection{Contact with live animals during veterinary curriculum}

In the past 18 months, have you experienced or been in a situation where you were at risk of exposure to animal urine (eg. dealing with, handling or being around live animals in a manner that you could have come in contact with urine, dead animals in anatomy or post mortem, collection or test of urine samples, reproductive tract manipulations or treatment, urinary tract surgery, etc.) or exposure where you may have been in contact with urine but not directly hands-on the animal (e.g. farm animal mustering, milking cows etc.)?

\section{YES $\square$ Complete table below \\ NO $\square$ Skip to Section 3.}

\begin{tabular}{|l|l|l|l|}
\hline Animal type & Exposure & Number of animals & $\begin{array}{l}\text { How many times in } \\
\text { past 18 months? }\end{array}$ \\
\hline Beef cattle & Yes $\square$ No $\square$ & & _ times \\
\hline Dairy cattle & Yes $\square$ No $\square$ & & times \\
\hline Sheep & Yes $\square$ No $\square$ & & $\ldots$ times \\
\hline Deer & Yes $\square$ No $\square$ & & times \\
\hline Horse & Yes $\square$ No $\square$ & & times \\
\hline
\end{tabular}




\begin{tabular}{|l|l|l|l|}
\hline Goat & Yes $\square$ No $\square$ & & - times \\
\hline Dogs & Yes $\square$ No $\square$ & & $\ldots$ times \\
\hline Cats & Yes $\square$ No $\square$ & & $\ldots$ times \\
\hline $\begin{array}{l}\text { Laboratory animals (i.e. rabbits, } \\
\text { mice) }\end{array}$ & Yes $\square$ No $\square$ & & Nimes \\
\hline \begin{tabular}{l} 
Other animals (please state) \\
\hline
\end{tabular} & Yes $\square$ times \\
\hline
\end{tabular}

\section{Exposure outside the veterinary curriculum}

This section asks about your potential exposure to the risk of leptospirosis from each of the animal species you may have been in contact with other than during the veterinary curriculum, i.e. during your personal life.

3.1 Own or have frequent contact with live animals

Do you own or have frequent contact (at least weekly) with live animals outside your veterinary curriculum?

YES $\square$ Please specify the animal types

NO $\square$ Skip to Exposure during personal life table 3.2

\begin{tabular}{|l|l|}
\hline Animal type & Own or have frequent contact \\
\hline Dogs, cats & Yes $\square$ No $\square$ \\
\hline Rabbits, guinea pigs or other small mammals & Yes $\square$ No $\square$ \\
\hline Horses & Yes $\square \quad$ No $\square$ \\
\hline Cattle, sheep, goats, deer, pigs & Yes $\square \quad$ No $\square$ \\
\hline Birds & Yes $\square$ No $\square$ \\
\hline Other & Yes $\square$ No $\square$ \\
\hline
\end{tabular}




\subsection{Contact with live animals at home, friend's or family's house}

Over the past 18 months, have you had regular contact (at least weekly) with animals outside your veterinary curriculum (at home, friend's or family's house) that may lead to exposure or risk of exposure to animal urine, for example, emptying your cat's litter tray or assisting your ewe's with lambing?

YES $\square$ Complete table below

NO $\square$ Skip to Wildlife table $\mathbf{3 . 3}$

\begin{tabular}{|l|l|l|l|}
\hline Animal type & Exposure & Number of animals & $\begin{array}{l}\text { How many times in } \\
\text { past 18 months? }\end{array}$ \\
\hline Beef cattle & Yes $\square$ No $\square$ & & times \\
\hline Dairy cattle & Yes $\square$ No $\square$ & & times \\
\hline Sheep & Yes $\square$ No $\square$ & & times \\
\hline Goats & Yes $\square$ No $\square$ & & times \\
\hline Deer & Yes $\square$ No $\square$ & & times \\
\hline Pigs & Yes $\square$ No $\square$ & & times \\
\hline Dogs & Yes $\square$ No $\square$ & & times \\
\hline $\begin{array}{l}\text { Cats } \\
\text { Yes } \square \text { No } \square\end{array}$ & Yes $\square$ No $\square$ & & times \\
\hline $\begin{array}{l}\text { Other_ } \\
\text { Did you have contact } \\
\text { with any of these } \\
\text { animals overseas? }\end{array}$ & Yes $\square$ No $\square$ If yes, specify country(ies) & \\
\hline
\end{tabular}

\subsection{Contact with wildlife animals}

Over the past 18 months, have you seen rats, mice, possums, rabbits or hedge hogs at where you were living (house, garden, surrounding fields)?

YES $\square$ Complete table below

NO $\square$ Skip to Home slaughter table 3.4 


\begin{tabular}{|l|l|}
\hline How many times in past 18 months? & \multicolumn{2}{|c|}{ times } \\
\hline $\begin{array}{l}\text { Have you set trapped or poisoned for } \\
\text { these animals at home? }\end{array}$ & Yes $\square \quad$ No $\square \quad$ Don't know $\square$ \\
\hline $\begin{array}{l}\text { Have you had contact with wildlife } \\
\text { animals overseas? }\end{array}$ & No $\square \quad$ Yes $\square \quad$ If yes, specify country(ies) \\
\hline
\end{tabular}

\section{$3.4 \quad$ Home slaughter}

Did you home-slaughter or have you helped with home slaughtering any animals in the past 18 months?

YES $\square$ Complete table below

NO $\square$ Skip to outdoor exposures table 3.5

\begin{tabular}{|l|l|l|l|}
\hline $\begin{array}{l}\text { Animal } \\
\text { type }\end{array}$ & $\begin{array}{l}\text { How many times } \\
\text { in past 18 } \\
\text { months? }\end{array}$ & $\begin{array}{l}\text { Number of animals } \\
\text { slaughtered? }\end{array}$ & When was the last time? \\
\hline Cattle & times & & \\
\hline Sheep & times & & \\
\hline Goats & times & & \\
\hline Deer & times & & \\
\hline Pigs & times & & \\
\hline
\end{tabular}

\subsection{Outdoor exposures}

\subsubsection{Hunting/Trapping exposures}

Have you been hunting in the past 18 months?

YES $\square$ Complete table below

NO $\square$ Skip to Other Outdoors exposure table 3.5.2 


\begin{tabular}{|l|l|l|l|}
\hline Animals hunted & $\begin{array}{l}\text { How many shot or } \\
\text { trapped }\end{array}$ & $\begin{array}{l}\text { When was the last } \\
\text { time? }\end{array}$ & $\begin{array}{l}\text { Animal butchered (by } \\
\text { yourself)? }\end{array}$ \\
\hline Deer & & & \\
\hline Wild pig & & & \\
\hline Small game * & & & \\
\hline Goats & & & \\
\hline Other & & & \\
\hline & & & \\
\hline
\end{tabular}

* e.g. ducks, other birds, possums, rabbits, hares...

\subsubsection{Other Outdoor exposure}

Over the past 18 months, have you done outdoor activities where you were exposed to fresh water?

YES $\square$ Complete table below

NO $\square$ Skip to Flooding 3.6

\begin{tabular}{|l|c|l|l|}
\hline $\begin{array}{l}\text { Outdoor activities fresh } \\
\text { water }\end{array}$ & $\begin{array}{l}\text { How many times in } \\
\text { past 18 months? }\end{array}$ & $\begin{array}{l}\text { When was the last } \\
\text { time? }\end{array}$ & Region? \\
\hline $\begin{array}{l}\text { Camping beside } \\
\text { lakes/rivers }\end{array}$ & times & & \\
\hline $\begin{array}{l}\text { Water sports in lakes/rivers } \\
\text { e.g. swimming, boating, } \\
\text { windsurfing, endurance } \\
\text { events }\end{array}$ & times & & \\
\hline Fresh water fishing & Nimes & & \\
\hline $\begin{array}{l}\text { Did you do any of these } \\
\text { activities overseas? }\end{array}$ & No $\square$ Yes $\square$ If yes, specify country(ies) & \\
\hline
\end{tabular}

\subsection{Flooding}

Over the past 18 months has where you were living been flooded?

YES $\square$ When the last time? (please state)

NO $\square$ Skip to Previous illness. 


\section{Previous illness}

Have you ever been diagnosed with Leptospirosis by a medical practitioner?

\section{YES $\square$ Complete Leptospirosis table 4.1 NO / Don't know $\square$ Skip to Other illness table 4.2}

\subsection{Leptospirosis (confirmed or suspected)}

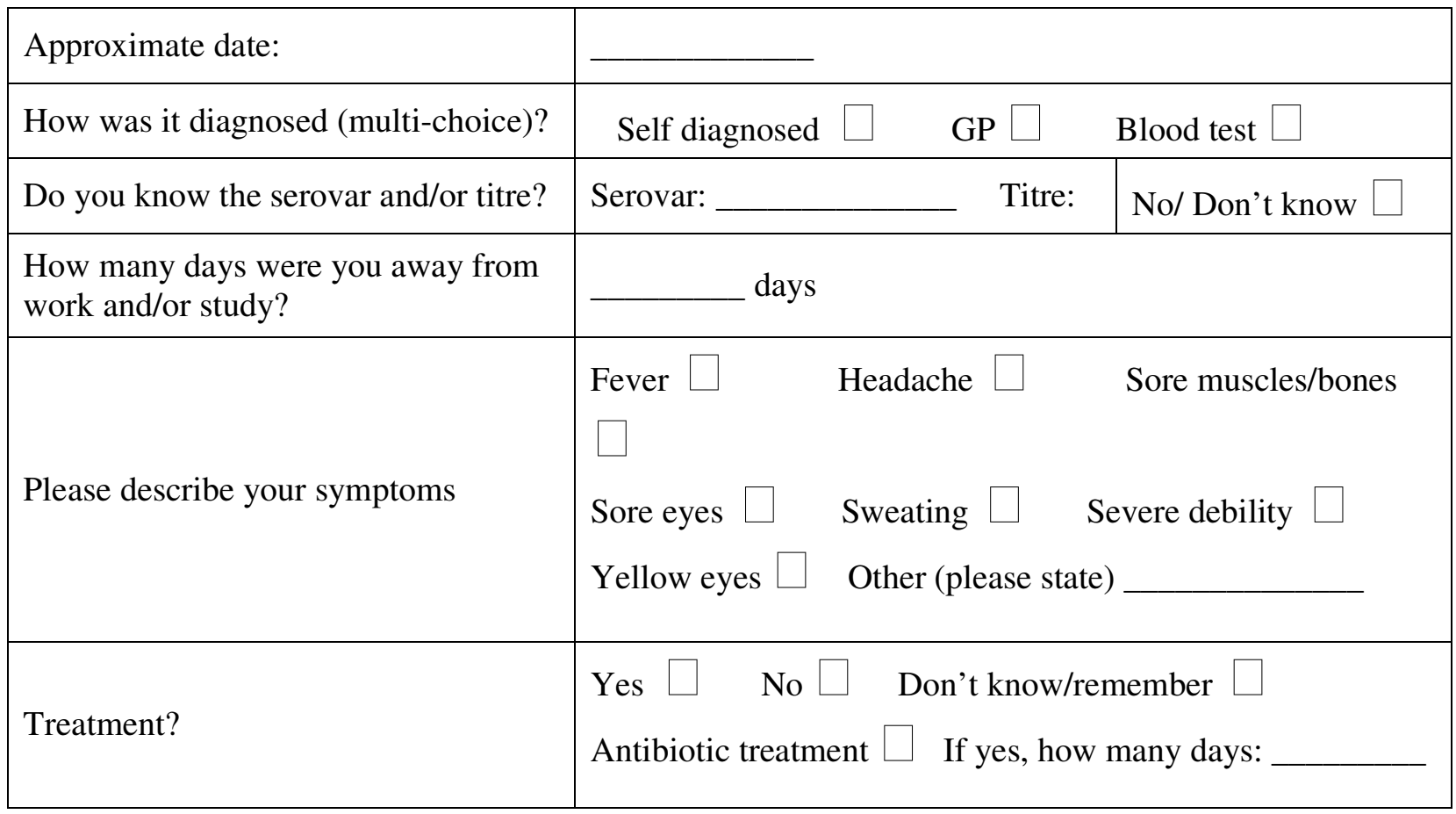




\subsection{Other Illness}

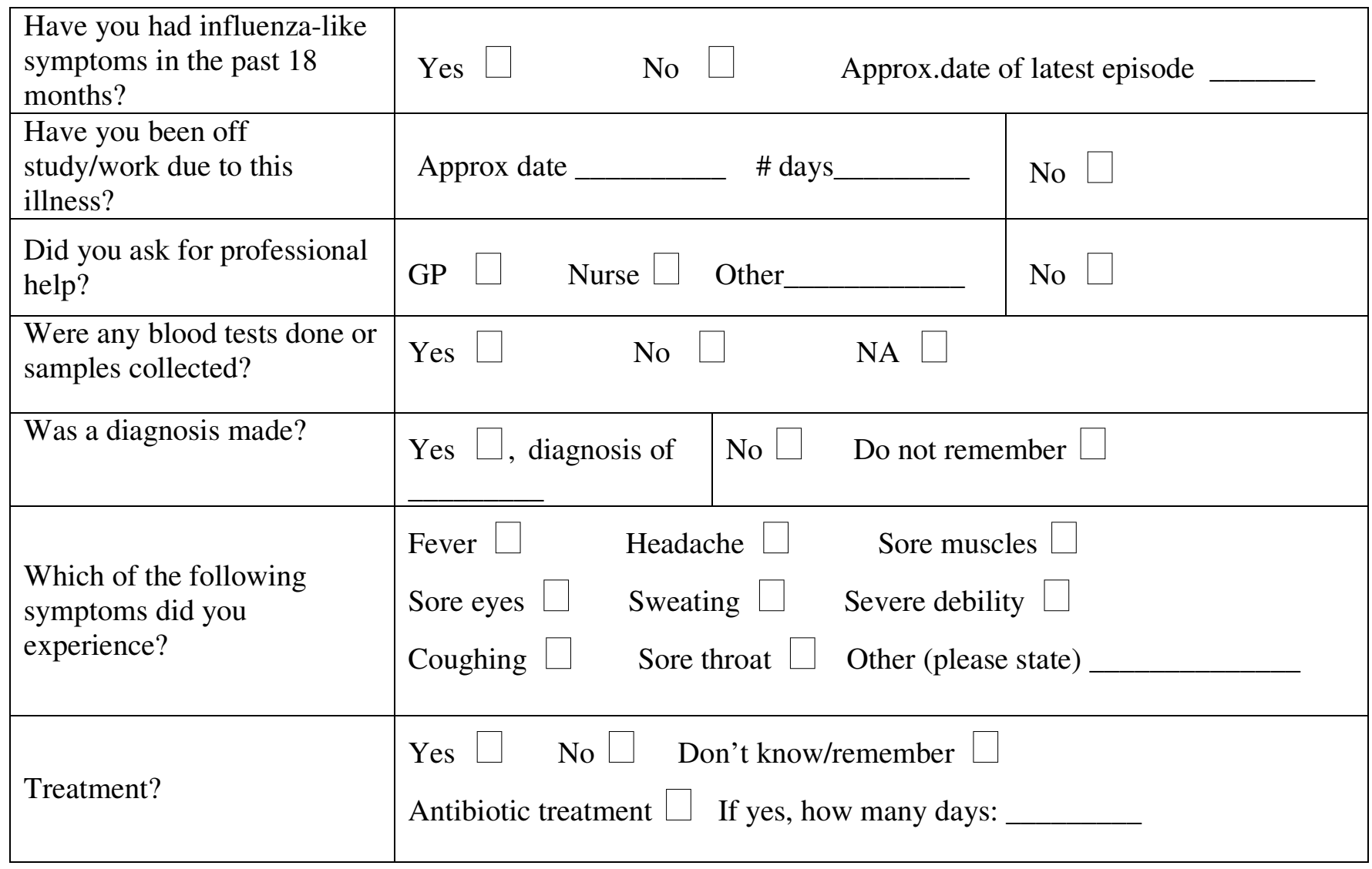

\section{- Comments}

Thank you so much for your participating in this survey. Please feel free to comment on your experience with leptospirosis or about this questionnaire. 